\title{
Spectral Filter Optimization for the Recovery of Parameters Which Describe Human Skin
}

\author{
Stephen J. Preece and Ela Claridge
}

\begin{abstract}
This paper presents a method for finding spectral filters that minimize the error associated with histological parameters characterizing normal skin tissue. These parameters can be recovered from digital images of the skin using a physics-based model of skin coloration. The relationship between the image data and histological parameter values is defined as a mapping function from the image space to the parameter space. The accuracy of this function is determined by the choice of optical filters. An optimization criterion for finding the optimal filters is defined by combing methodology from differential geometry with statistical error analysis. It is shown that the magnitude of errors associated with the optimal filters is typically half of that for typical RGB filters on a three-parameter model of human skin coloration. Finally, other medical image applications are identified to which this generic methodology could be applied.
\end{abstract}

Index Terms-Color, image analysis, spectral filters, optimization, skin color, medical imaging.

\section{INTRODUCTION}

$\mathrm{D}$ ESPITE considerable variability in skin hues across different human groups, the skin color depends primarily on three histological variables: The amount of melanin pigment, the amount of blood-borne hemoglobin pigments, and the thickness and structure of collagen in superficial skin layers [1], [2]. The idea of describing the entire range of skin coloration using only three parameters is very attractive. Such concise parameterization can be used in many different application areas, from realistic rendering of skin tones in computer graphics and multimedia (e.g., [3]), to clinical and cosmetic applications which investigate the state of health of the human skin (e.g., [4]).

Our earlier work [2], [5] developed a novel method for recovering histological skin parameters from image data using a physics-based model of image formation. An outline of that method is given in Section 3. This paper concentrates on the problem of the accuracy of tissue parametrization, which is approached by seeking to minimize the error in parameter recovery. The spectral filter optimization is a mechanism through which error minimization is accomplished.

Typically, optical filters are based on the RGB system or some otherCIE-based system. These are clearly the best choice if a measure of color, as perceived by humans, is required. However, they may not be the optimum choice for an imaging system that is to recover specific parameters from imaged data. As discussed by Angelopoulou et al. [3], the acquisition of data through RGB filters is equivalent to "projecting infinitely dimensional color space to a three-dimensional space. This is a many-to-one projection and results in colors with different spectral distributions giving the same RGB response." This is

- S.J. Preece is with Astron Clinica, The Mount, Toft, Cambridge CB3 7RY UK. E-mail: sjp@cs.bham.ac.uk.

- E. Claridge is with the School of Computer Science, The University of Birmingham, Birmingham B15 2TT UK.

E-mail:E.Claridge@cs.bham.ac.uk.

Manuscript received 21 Aug. 2002; revised 28 July 2003; accepted 2 Jan. 2004. Recommended for acceptance by R. Chellappa.

For information on obtaining reprints of this article, please send e-mail to: tpami@computer.org, and reference IEEECS Log Number 117158. known as metamerism. Even if metamerism does not occur the projection may still result in similar RGB values for spectra corresponding to different skin characteristics. This may be a problem in biomedical applications that require the ability to depict subtle variations in the quantities of the skin tissue components. With the optimal filters, a given imaging system is guaranteed to produce images that will yield the specific histological parameters with minimum error.

The paper is structured as follows: Section 2 provides a review of the related work. The following section outlines the overall method for skin parameter recovery through the model of skin coloration. A detailed mathematical formulation of the problem is then outlined and optimization criteria to determine appropriate optical filters are defined. A threeparameter model of human skin is presented in the subsequent section. It is shown that the objectively defined set of filters performs better than a standard RGB system. Conclusions are then drawn and other medical image applications are identified to which the methodology could be applied.

\section{Review of the Related Work}

The three related areas of work include the modeling of skin appearance, the estimation of skin parameters from color images, and methods for optimal filter selection in context of image interpretation.

The problem of skin color modeling has been studied extensively within the computer vision and graphics communities. In the applications requiring realistic skin color renditions, the most common approach is to identify a small number of basis functions from which skin spectra and thus skin colors can be reconstructed. For example, Angelopoulou et al. [3] used Gaussians and Gaussian derivatives as the basis functions and were able to produce faithful reconstructions of skin spectra with a linear mixture of five and four functions, respectively. Tsumura et al. [6] used independent component analysis to identify vectors in RGB space corresponding to change in the amount of the pigments melanin and hemoglobin. Their technique was able to simulate specific changes in skin colors; for example, tanning, reddening, and enhancement of skin blemishes by 
changing the parameters corresponding to quantities of the two pigments.

Similar techniques were developed in image analysis to understand and identify skin appearance in uncalibrated color images. Based on the analysis of illuminant chromaticities by Finlayson and Schaffer [7], Stoerring and Granum [8] developed a physics-based model of skin coloration under different illuminants. Through eigenspace analysis they estimated the distribution of skin colors under different illuminants and successfully applied the method to segmentation of skin colors. Ohtsuki and Healey [9] modeled reflectance values of the skin as a function of melanin and blood and combined it with a 3D geometric model of the face. Using likelihood estimation, they were able to detect facial features such as the eyes and mouth in faces imaged in arbitrary poses and under arbitrary light conditions. In general, much research has been carried out into fundamental aspects of illumination invariant detection of skin colors in complex scenes; however, this topic is outside the scope of this paper and is not reviewed here further.

In line with several approaches described above (e.g., [3] and [6]), our work uses a generative (forward) model of skin reflectance. However, by incorporating a nonlinear component arising from scatter in the papillary dermis and using physics-based light transport equations [10], [11] rather than statistical approaches, our forward model is likely to be more accurate.

In another group of applications, which is the focus of this work, the aim is to recover skin parameters from color images. This ability is required for in vivo studies and the diagnosis of skin disorders and for noninvasive monitoring and evaluation of the effects of therapeutic or cosmetic treatments. The process of parameter recovery is more complicated than in the modeling of the skin appearance. If physics-based methods are used, the inverse solution of the light transport model is difficult to obtain algebraically due to the presence of nonlinearities in the model [12]. When using statistical approaches (e.g., [6]) there is no guarantee that the parametrization obtained is compatible with histology. This is in contrast with our approach where the histologically compatible parameterization is explicitly incorporated.

The method for selection of the optimal filters is, in the context of this work, a part of the parameter recovery process. However, it can also be viewed in the context of hyperspectral imaging where an objective is typically to classify objects and features by exploiting differences in their spectral characteristics. The optimization criteria are usually based on general statistical and mathematical theories. For example, Chang et al. [13] developed spectral band selection methods based on principal component analysis and on Fisher's discriminant analysis for remote sensing image classification. Shen and Basset [14] used an information-based theory to determine the optimal wavebands for differentiating a large number of objects with well-defined spectral characteristics. Similarly, Karlhom and Renhorn [15] used the results from the detection theory to select spectral bands optimized for discrimination between specific classes of objects in a cluttered background. Price [16] selected spectral bands based on their physical significance.

In general, the criteria used in hyperspectral imaging lead to the clustering of points in the filter (feature) space. In contrast, the error minimization criterion used in this work encourages maximum dispersion of the feature points corresponding to a given parameter, leading to the increase in accuracy in parameter recovery.

\section{Skin Parameter Recovery}

The selection of optimal filters is a part of a broader method for the recovery of tissue parameters from tissue image data, originally developed by Cotton and Claridge [2], [17]. To provide an appropriate context, a brief description of the overall method is given. It consists of two steps, the modeling step that is carried out only once, and the parameter recovery step that is carried out for each image instance.

The purpose of the modeling step is to establish a systematic relationship between tissue parameters and image values. The underpinning assumption is that histological variations in the imaged skin can be described by a small number of parameters. For each combination of the parameter values, a suitable model of light transport computes the corresponding spectra. These spectra are then converted to discrete image vectors by convolving them with a set of spectral (bandpass) filters, typically RGB. In this way, for each parameter vector, there is a corresponding vector of image values. The mapping must be unique and we describe how this condition is tested in Section 5. A discrete computational representation relating the parameter and the image vectors is called the model of skin coloration.

In the parameter recovery step, an image acquired through the same set of filters as used to develop the model is scanned pixel by pixel. For each image vector, a corresponding parameter vector is obtained from the model of skin coloration. The value of each parameter is then displayed in a separate gray-scale image that shows how the magnitude of each parameter varies spatially. These images are referred to as parametric maps. The information displayed in such maps is proving to be immensely valuable to clinicians in their assessment of pigmented skin lesions and in the diagnosis of melanoma, a skin cancer [4]. Fig. 1 shows a color image of a skin lesion and three parametric maps corresponding to melanin, blood, and the thickness of the papillary dermis.

A clinical system developed on the basis of these ideas, the SIAscope, images small areas of skin via a transparent surface placed in contact with the imaged tissue. An arrangement of crossed polarization filters is employed to eliminate the specular component of reflection. Images acquired using this system can be assumed to vary in only two spatial dimensions and to contain no surface reflections.

\section{Formulation of the Problem}

The technique is first formulated for a model of imaged tissue which requires $K$ parameters to describe all histological variation. Later, in Section 6, the methodology is demonstrated on human skin for the case where $K=3$.

For a $K$-parameter model the parameter vector is defined as

$$
\mathbf{p}=\left\langle p_{1}, p_{2}, \ldots, p_{K}\right\rangle \quad \mathbf{p} \in \mathbf{P},
$$

where $\mathbf{P}$ defines the space of all possible parameter variations and, thus, variations in skin histology. The ultimate objective is to recover a parameter vector from a vector of image values 


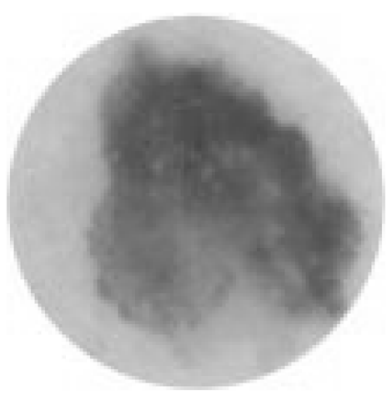

(a)

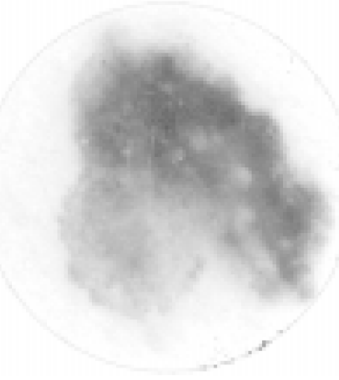

(b)

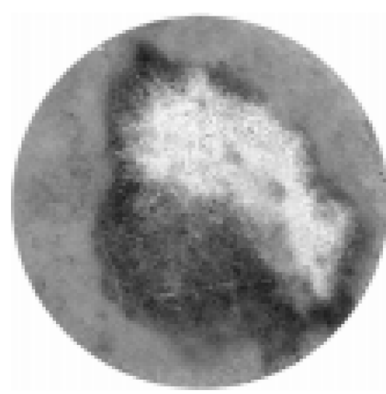

(c)

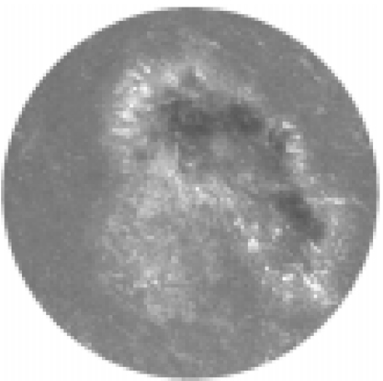

(d)

Fig. 1. Parametric maps for a pigmented skin lesion: (a) original color image, (b) parametric map showing melanin concentration, and (c) parametric map showing blood concentration. Dark hues in the lower part of the lesion are due to high blood concentration. This is impossible to determine from the color image alone. The blood map also shows a blood deprived region (light hues) in the upper part of the lesion which is impossible to see from the color image because of the masking effect of the melanin. (d) Parametric map showing collagen concentration. The parametric maps reveal internal composition of the lesion and help in diagnosis.

at every point in a given image. To achieve this, it is first necessary to consider the forward problem of obtaining a vector of image values from a given parameter vector.

A reflectance spectrum can be described by the vector in $M$-dimensional reflectance space

$$
\boldsymbol{\lambda}=\left\langle\lambda_{1}, \lambda_{2}, \ldots, \lambda_{M}\right\rangle \quad \boldsymbol{\lambda} \in \boldsymbol{\Lambda},
$$

where the space $\boldsymbol{\Lambda}$ defines all possible spectral reflectance functions. The mapping $a$ defined as

$$
a: \mathbf{P} \longrightarrow \boldsymbol{\Lambda}
$$

is introduced to denote the mapping from parameter space to reflectance space. This mapping gives the spectral reflectance for a given parameter vector $\mathbf{p}$. Such a mapping can be achieved by using a mathematical model that takes as input the parameter vector and gives a corresponding reflectance spectrum. A suitable physics-based model of light propagation is required for this purpose, such as the Monte Carlo method [10] or the Kubelka Munk approximation [11]. Mapping (3) must be defined across the whole of parameter space, thus defining every possible spectral reflectance function.

A digital camera with $N$ optical filters records a $N$-dimensional image vector at each pixel. The image vector is given as

$$
\mathbf{i}=\left\langle i_{1}, i_{2}, \ldots, i_{N}\right\rangle \quad \mathbf{i} \in \mathbf{I},
$$

where I describes the space of all possible image values, referred to as image space. The process of image acquisition can be considered as the projection of points in reflectance space to points in image space. This mapping is performed by the function

$$
b: \Lambda \longrightarrow \mathbf{I} .
$$

In continuous form, the mapping $b$ can be written as

$$
i_{n}=\int E(\lambda) S(\lambda) R^{n}(\lambda) d \lambda,
$$

where $i_{n}$ is the $n$th component of the image vector $\mathbf{i}, \lambda$ denotes wavelength, $E(\lambda)$ is the illumination, $S(\lambda)$ the object spectral reflectance and $R^{n}(\lambda)$ the response of the $n$th optical filter. In discrete form, this becomes

$$
i_{n}=\sum_{m=1}^{M} R_{m}^{n} \lambda_{m}
$$

where $\lambda_{m}$ is the $m$ th element of the discretized reflectance spectrum, given by the product $E(\lambda) S(\lambda)$. The positive weights at each wavelength are given by $R_{m}^{n}$, thus defining each filter response function. A digital camera effectively performs mapping $b$, projecting points from a large-dimensional space (reflectance space) to a small-dimensional space (image space). With such a mapping, there will be a substantial loss of information. Provided certain conditions are fulfilled, it is possible to define the mapping in such a way that relatively accurate information regarding the original parameter values can still be recovered from image data. These conditions are discussed in the following section.

Most current image acquisition systems use an RGB system of filters. Although this defines a potential mapping $b$, it may not be the best mapping with which to recover parameter values in specific cases, such as from skin image data.

The function $f$ defined as

$$
f=a \circ b \quad f: \mathbf{P} \longrightarrow \mathbf{I}
$$

represents the two-stage mapping from parameter space to reflectance space to image space. Provided it is possible to obtain a spectrum for any given parameter vector, mapping (8) can be performed across the whole of parameter space. The inverse of function $f$ defined as

$$
g: \mathbf{I} \longrightarrow \mathbf{P}
$$

maps from the space of image values directly to parameter space. The objective of this work, restated more formally, is to find the optimum $g$ which maximizes the accuracy of parameter recovery. Before a detailed discussion of this mapping is presented, it is important to emphasize that the form of the function $f$ and, thus, $g$ depends on the mappings $a$ and $b$. Whereas mapping $a$ is fixed, mapping $b$ can be determined by the choice of optical filters.

\section{Defining an Optimum Mapping Function}

\subsection{Uniqueness of Mapping}

Any mapping function, $g$, which is to map from the space of image values (I-space) to parameter space ( $\mathbf{P}$-space) must be one-to-one. That is, for every point in $\mathbf{P}$-space, there must 
be a corresponding unique point in I-space and vice versa. If this is not the case, ambiguity will arise as it could be possible to recover more than one set of parameter values from a given vector of image values.

The uniqueness of mapping is determined by using analytical tools of differential geometry [18]. In conformance with this domain, the mapping function $\mathbf{f}$ is defined as a vector valued function of a vector variable

$$
\mathbf{i}=\mathbf{f}(\mathbf{p}) \text {. }
$$

To establish whether the function $\mathbf{f}$ is one-to-one the behavior of the determinant of the Jacobian matrix is considered. This matrix is defined as

$$
\mathbf{J}=\left(\begin{array}{cccc}
\frac{\partial i_{1}}{\partial p_{1}} & \frac{\partial i_{1}}{\partial p_{2}} & \cdots & \frac{\partial i_{1}}{\partial p_{K}} \\
\cdots & \cdots & \cdots & \cdots \\
\frac{\partial i_{N}}{\partial p_{1}} & \frac{\partial i_{N}}{\partial p_{2}} & \cdots & \frac{\partial i_{N}}{\partial p_{K}}
\end{array}\right)
$$

At this point, we note that a determinant can only be defined for a square matrix, thus the analysis presented in this paper is limited to the case where $N=K$. The inverse function theorem [18] states that, if the determinant of the Jacobian matrix is nonzero at a point $\mathbf{p}=\mathbf{p}^{0}$ in $\mathbf{P}$-space, then there exists a neighborhood around $\mathbf{p}^{\mathbf{0}}$, where the function $\mathbf{f}$ can be approximated linearly. Points within this neighborhood will therefore map, under a one-to-one mapping, to points in a corresponding neighborhood within I-space, centered on the point $\mathbf{q}=\mathbf{q}^{0}$. If the Jacobian matrix is strictly positive or strictly negative throughout the whole of $\mathbf{P}$-space, then the function $\mathbf{f}$ is one-to-one everywhere. This condition can be viewed as an extension of the one-dimensional case where the absence of a zero derivative ensures no turning points and, thus, the uniqueness of function over a given range.

Within the neighborhood where the function $\mathbf{f}$ can be approximated linearly it is possible to express the inverse function $\mathrm{g}$ as

$$
d \mathbf{p}=\mathbf{J}^{-1} d \mathbf{i},
$$

where $d \mathbf{p}=\mathbf{p}-\mathbf{p}^{0}$ and $d \mathbf{i}=\mathbf{i}-\mathbf{i}^{0}$. In long-hand form, $\mathbf{g}$ is given as

$$
\begin{aligned}
& d p_{1}=\frac{\partial p_{1}}{\partial i_{1}} d i_{1} \quad \frac{\partial p_{1}}{\partial i_{2}} d i_{2} \quad \cdots \quad \frac{\partial p_{1}}{\partial i_{K}} d i_{K} \\
& \begin{array}{cccccc}
\vdots & = & \vdots & \vdots & \vdots & \vdots \\
d p_{N} & = & \frac{\partial p_{N}}{\partial i_{1}} d i_{1} & \frac{\partial p_{N}}{\partial i_{2}} d i_{2} & \cdots & \frac{\partial p_{N}}{\partial i_{K}} d i_{K} .
\end{array}
\end{aligned}
$$

Expression (12) gives a linear approximation for each separate component of the parameter vector in terms of a given vector of image values and a matrix of derivatives that is obtained by inverting the Jacobian matrix. This linearized expression in only valid within a small neighborhood of $\mathbf{P}$-space. Thus, it is necessary to construct a piecewise continuous approximation for the function $\mathrm{g}$ at discrete intervals across parameter space. Once this has been achieved, it will be possible to recover a vector of parameter values for all possible vectors of image values.

\subsection{Error of Mapping}

If the mapping were error-free, any set of filters that meets the uniqueness criterion above could be used to recover the parameter vector. However, due to experimental uncertainty in the image values measured using a digital camera, there is uncertainty in the vector of parameter values recovered using a given function $\mathrm{g}$. The effect of this error is considered with the aim of defining an optimization criterion for determining the optimum filters to be used in image acquisition. The particular approach is to examine how camera noise will propagate into a corresponding error in the parameter vector recovered from image data.

A camera measurement can be treated in the same way as a standard experimental measurement which is normally distributed about the measured value with a known standard deviation or experimental error [19]. The standard deviation of the camera noise, commonly available from a technical specification data sheet, is defined as $\sigma^{\text {cam }}$. Each component of the function $\mathrm{g}$ must now be considered a function of random variables, namely, the normally distributed camera measurements. By applying the appropriate statistical analysis, see Kendall and Stuart [20] for details, it can be shown that the error in the $k$ th parameter is given as

$$
\sigma_{g_{k}}=\sigma^{c a m} \sqrt{\sum_{n=1}^{N}\left(\frac{\partial p_{k}}{\partial i_{n}}\right)^{2}} .
$$

By applying (14) for each parameter, it is possible to obtain a vector of errors that describe the uncertainty in each parameter at a given point in parameter space. The components of this vector correspond to the standard deviations of each of the parameters and, thus, define normal distributions for each of the recovered parameters.

\subsection{Finding an Optimal Set of Filters}

It was explained earlier that the Jacobian matrix can only be assumed to be constant within a small neighborhood of parameter space. Thus, it is necessary to discretize parameter space in suitably small intervals. At each point within a discretized space, it is possible to obtain a value for the error in each parameter. With this measure of accuracy, it is possible to define an optimization criterion to find the optimum mapping $f$ defining the filters for image acquisition.

An algorithm for the implementation of the proposed methodology is given as follows:

1. Establish a suitable search space from a parameterization of appropriate filter response functions.

2. For a given set of filter response functions, calculate the vector of image values for each point within a discretized parameter space.

3. For each point, check that the Jacobian is either strictly positive or strictly negative across the whole of parameter space. If this condition is held, then compute the inverse of the Jacobian matrix. If not, then return to Step 1 and define a new set of filter response functions.

4. Using (14), calculate the error in recovery of each parameter across the whole of parameter space and use this measure to define a suitable optimization criterion.

5. Repeat Steps 2-4 with some technique which enables an optimum set of filter response functions to be determined.

To define an appropriate search space, it is necessary to parameterize mapping $b$ which defines the filters used for image acquisition. This mapping is defined by the 


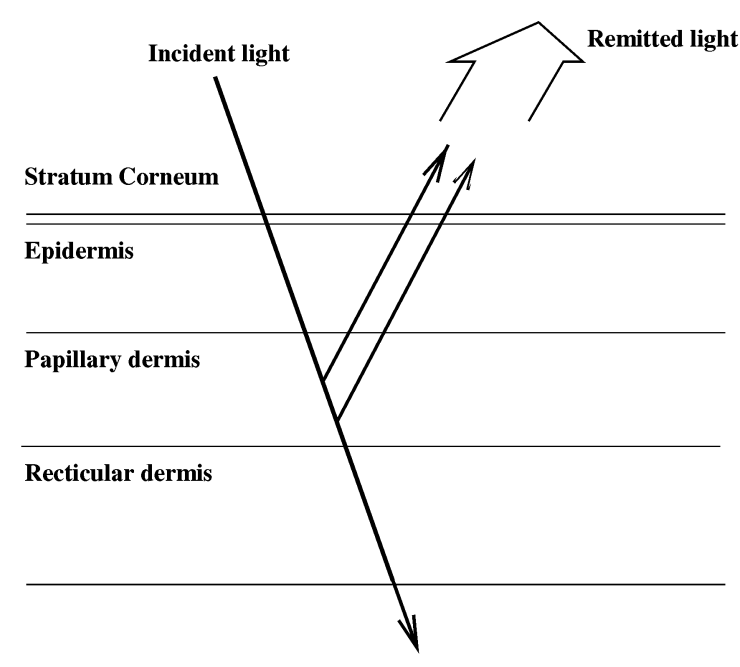

Fig. 2. Structure of normal skin.

positive $N \times M$ matrix $R_{m}^{n}$, given in (7). Coefficients $R_{m}^{n}$, $m=1, \ldots, M$, specify the shape of $n$th filter. For practical reasons, we require that the parameterization is based on a typical optical filter. As an example, the position of the central wavelength and a measure of width could be used to define a Gaussian shaped filter.

An optimization method should search the whole space of possible filter response functions using the optimization criterion outlined above.

\section{Three-Parameter Model of the NORMAL SKIN}

\subsection{Prediction of Spectral Reflectance}

The model of spectral reflectance of the skin employed in this work was originally developed by Cotton and Claridge [2]. It is summarized below. Skin is considered the fourlayer structure depicted in Fig. 2. All histological variation can be characterized by changes in concentration of melanin and blood, and in papillary dermal thickness, thus defining a three-dimensional parameter space. Although not absorbing any radiation, the stratum corneum scatters the incoming light in all directions. Light that leaves this layer and enters epidermis can thus be considered diffuse. In the epidermis, light is absorbed by the pigment melanin. The absorption at each wavelength can be calculated using the Lambert-Beer law

$$
\theta(\lambda)=e^{-c m(\lambda)},
$$

where $m(\lambda)$ is the melanin extinction coefficient and $c$ is the concentration of melanin. There is very little forwarddirected scattering in the epidermis and all light not absorbed by melanin can be considered to pass into the papillary dermis. There, the light is both scattered and absorbed. The absorption results from the presence of blood and scattering from the underlying collagen structure. The simple KubelkaMunk light transport theory [11] can be used to calculate the fraction of light remitted from the papillary dermis. Mathematically, this is given as

$$
R(\lambda)=\frac{\left(1-\beta(\lambda)^{2}\right)\left(e^{K(\lambda) d}-e^{-K(\lambda) d}\right)}{(1+\beta(\lambda))^{2} e^{K(\lambda) d}-\left(1-\beta(\lambda)^{2}\right) e^{-K(\lambda) d}},
$$

where

$$
K(\lambda)=\sqrt{k(\lambda)(k(\lambda)+2 s(\lambda))} \quad \text { and } \quad \beta(\lambda)=\sqrt{\frac{k(\lambda)}{k(\lambda)+2 s(\lambda)}} .
$$

$\lambda$ is the wavelength and $d$ is the thickness of the papillary dermis. The parameter $k(\lambda)$ characterizes the absorption of the tissue and is calculated from the sum of the absorption of the structural tissue and that of the blood born pigments, hemoglobin, and oxyhemoglobin. The latter is calculated from the product of the blood concentration and the specific extinction coefficients of hemoglobin and oxyhemoglobin [1]. The parameter $s(\lambda)$ characterizes the scatter which occurs due to the collagen fibers forming a structural part of the papillary dermis [21]. Any light transmitted through papillary dermis and reaching the reticular dermis is scattered forward and, thus, does not make a contribution to the remitted fraction. The light remitted from the papillary dermis travels back through the epidermal layer where it is again partially absorbed by melanin. Using this light transport model, it is possible to obtain the remitted intensity at a number of wavelengths and, thus, construct a remitted spectrum, $S(\lambda)$. This spectrum is a function of the three-component parameter vector, that is, the concentration of melanin and blood and thickness of the papillary dermis. It is assumed that there is no spectral variation in the intensity of the illuminating light and thus $E(\lambda)=1$. A more detailed description of this model can be found in [2].

To carry out the optimization procedure developed in Section 5 , it is necessary to discretize parameter space. This is done at equal intervals to define $10 \times 10 \times 10$ points. For simplicity, concentration and thickness values will be denoted by a number between 1 and 10. Fig. 3a shows how the remitted spectrum changes as the melanin concentration is varied. With a change in melanin concentration, the intensity of the whole spectrum is seen to decrease, with a more pronounced change in the blue region. Fig. $3 \mathrm{~b}$ shows the spectral changes that correspond to changes in blood concentration. In this case, the largest change is observed in the green region, the resulting shape reflecting the two absorption maxima of oxyhemoglobin. Fig. $3 c$ shows the spectral changes which occur when the thickness of the papillary dermis is varied. The effect of this parameter is to reduce the intensity of the remitted spectrum above the wavelength $\lambda=450$.

\subsection{Obtaining an Optimum Mapping Function}

A parameterized form of $b$ is chosen to define a typical interference filter which is modeled as a square profile with Gaussian decay at each side. Two parameters are sufficient to specify this shape: The central wavelength and a full width half maximum (FWHM). Three filters are required to recover a three-valued parameter vector thus defining a sixdimensional search space.

The optimization procedure was implemented following the algorithm given in Section 5. Initially, the vector of image values was calculated for every point within the discretized parameter space for a given set of filter response functions. The derivative of each image value, with respect to each 


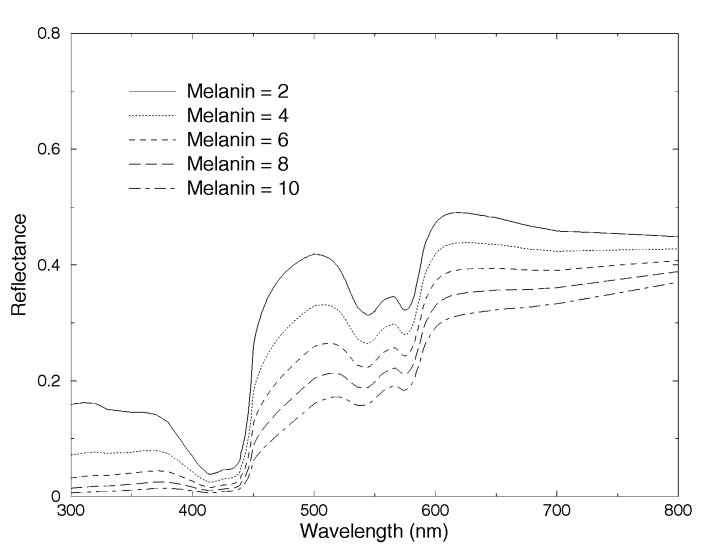

(a)

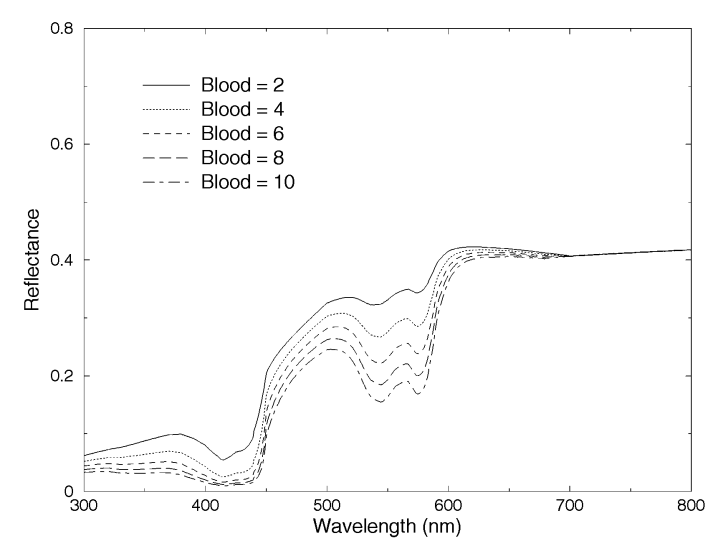

(b)

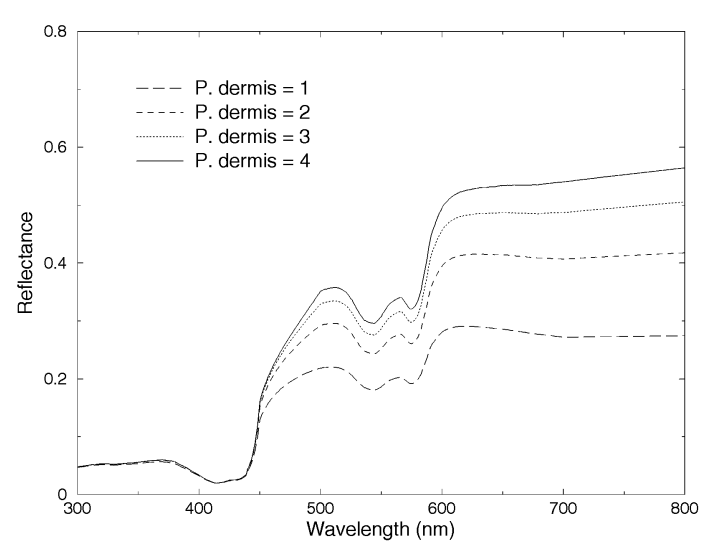

(c)

Fig. 3. (a) Remittance spectrum of normal human skin for varying melanin concentration with blood concentration at level 5. (b) Remittance spectrum of normal human skin for varying blood concentration with melanin concentration at level 5. (c) Remittance spectrum of normal human skin for varying thickness of papillary dermis with melanin and blood concentration both at level 5 .

parameter, was obtained at each point within discretized parameter space using three-point finite-difference approximations. The Jacobian matrix was then constructed at every point within parameter space, and providing its determinant was nonzero and of equal sign everywhere, the inverse was calculated. If this condition was violated, then a new set of filter response functions was defined.

If the uniqueness condition was fulfilled, then the error in each parameter was calculated using (14) at every point within a discretized parameter space. This error depends on the accuracy of the camera, $\sigma^{\text {cam }}$, which was taken to be two gray-scale levels in an 8-bit representation (2/255). A total error in each of the three parameters was obtained by summing the errors across parameter space and the accuracy taken to be the reciprocal of this quantity. The optimization criterion to be maximized was taken to be the sum of the three accuracies. This procedure was implemented in Matlab using a standard genetic algorithm (GA), although any optimization algorithm could have been used with an equal effect. Genetic algorithms belong to a class of stochastic optimization methods that normally suffer less from becoming trapped in local optima. In addition, the optimization criteria are very easy to specify as they do not depend on the specific structure of a problem. As the main focus of this paper is to present a methodology for determining an appropriate optimization criteria, a comparison of different optimization techniques is not included.

The GA was set out to search the space of available filter response functions. The boundaries of the search space were chosen such that the central wavelength was constrained to lie in the visible region (400nm-700nm) and such that the widths of the filters were allowed to vary from a FWHM of 25 to $100 \mathrm{~nm}$. Although it is now possible to engineer almost any shape of interference filter this corresponds to an economically viable range of such filters. Filter overlap was prohibited.

Equation (6) gives image values in terms of incident light, object spectral reflectance, and response of the $n$th filter. In practice, this value is also influenced by the camera gain that could potentially be different for each channel. For the purpose of this investigation, filter values were normalized against their white light value. Thus, the use of a wider filter did not necessarily improve the accuracy of parameter recovery and allowed the optimization method to determine the most appropriate spectral regions for measurement of histological parameters.

The shape of each filter will also be modified by the optics of the image acquisition system. For example, factors such as detector quantum efficiency and spectral transmission of the imaging lens will modify the response of each filter. As such factors vary slowly with wavelength, they can be considered constant across the wavelength range corresponding to a typical interference filter. By normalizing the filters by their white light values, the effect of these additional optical characteristics can be neglected.

\section{REsULts}

\subsection{Optimal Filters}

The results of the genetic algorithm consistently returned the filter definitions given in Table 1 (filters 1, 2, and 3). The table also lists the definitions of typical RGB filters.

\subsection{Errors in Parameter Recovery}

Table 2 characterizes relative errors computed via error propagation for the measurement of melanin, blood, and thickness of the papillary dermis. 
TABLE 1

Filter Definitions Returned by the Optimization Procedure and Definitions of Typical RGB Filters

\begin{tabular}{|c|c|c|}
\hline Filter & CW (nm) & FWHM (nm) \\
\hline 1 & 485 & 24 \\
\hline 2 & 560 & 14 \\
\hline 3 & 700 & 95 \\
\hline R & 650 & 25 \\
\hline G & 550 & 25 \\
\hline B & 450 & 25 \\
\hline
\end{tabular}

For each parameter the median, the mean, and standard deviation are given for the optimal filters and the RGB filters listed in Table 1. Graphs in Figs. 4, 5, and 6 show the behavior of the relative errors, for melanin, blood, and papillary dermis, respectively, as a function of concentration of melanin and blood. The magnitude of errors associated with the optimal filters is typically half of that for the RGB filters.

Plots in Fig. 7 show the relationship between the histological parameters and image values for the optimal filters and for the RGB filters. The dispersion of the parameter values with respect to the image values is much greater for the optimal filters, explaining why the parameters can be recovered with higher accuracy. These results clearly show the advantage of carefully tuning the spectral responses of the filters for the specific tissue components.

\subsection{Discussion of the Results}

It is interesting to analyze the choice of optimal filters by relating them to the graphs showing the spectral variability of the individual parameters (Fig. 3). A fairly broad filter centered at $700 \mathrm{~nm}$ will clearly capture variation in the
TABLE 2

Relative Errors of Parameter Recovery for Melanin, Blood, and Papillary Dermis

\begin{tabular}{|c|c|c|c|c|c|c|}
\hline \multirow{2}{*}{} & \multicolumn{2}{|c|}{ Melanin } & \multicolumn{2}{c|}{ Blood } & \multicolumn{2}{c|}{ Pap. Dermis } \\
\cline { 2 - 7 } & RGB & Opt & RGB & Opt & RGB & Opt \\
\hline Median & 15.75 & 8.49 & 18.00 & 10.65 & 7.81 & 4.29 \\
\hline Mean & 30.10 & 11.79 & 26.10 & 14.05 & 8.49 & 4.44 \\
\hline StDev & 36.67 & 10.72 & 20.23 & 10.29 & 3.40 & 0.75 \\
\hline
\end{tabular}

papillary dermis thickness. A filter centered at 560nm corresponds to the range of wavelengths where sensitivity to change in blood level is very high, e.g., the peak absorption for oxyhemoglobin is at $558 \mathrm{~nm}$. The first filter, at $485 \mathrm{~nm}$, coincides with the maximum variability related to melanin levels. On initial inspection, one might be tempted to assume that filters 1, 2, and 3, given in Table 1, are used to recover values of the parameters melanin, blood, and papillary dermal thickness independently. It is important to emphasize that this is not the case. Each of the histological parameters has some effect at every point across the whole visible spectrum. Thus, each filter, will to some degree, capture information on all three parameters. This multiple dependency is expressed mathematically by treating the mapping function (10) as a vector valued function of a $K$-dimensional vector variable. Such treatment is close to the real image acquisition where independent measurement of individual variables is only rarely possible.

\section{General Discussion}

The main contribution of this work is a method for finding optimal filters that minimize the error associated with the parameter values characterizing skin tissue. Although the filter optimization method described is a part of a broader

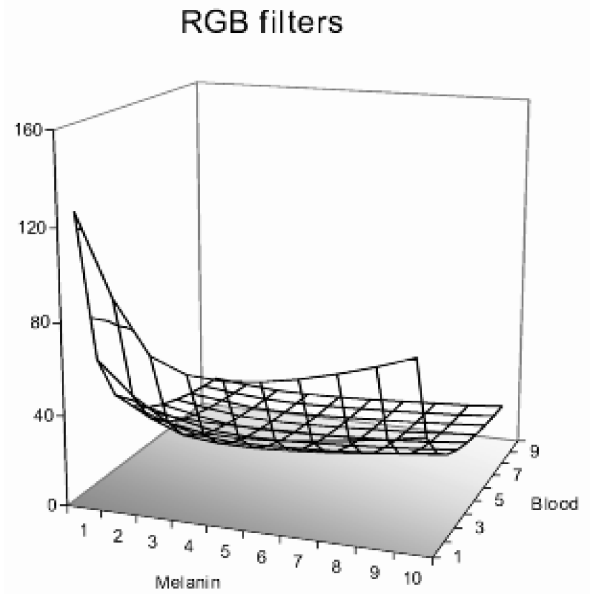

(a)

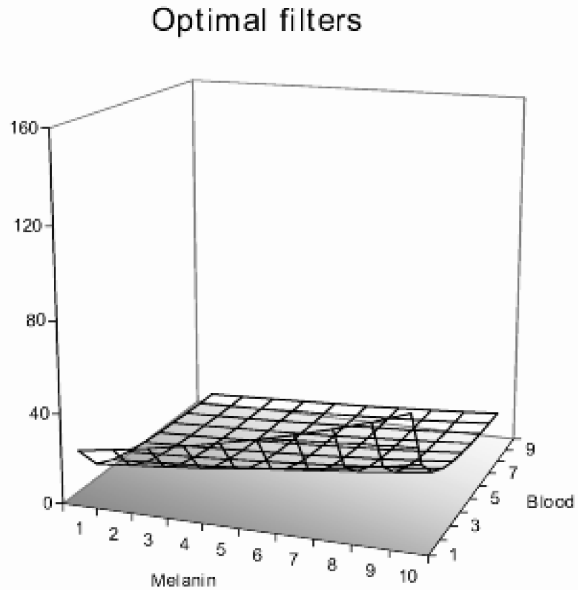

(b)

Fig. 4. Plot of the relative error in the recovery of blood concentration as a function of melanin and blood concentration, (a) for RGB filters and (b) for the optimal filters. 
RGB filters

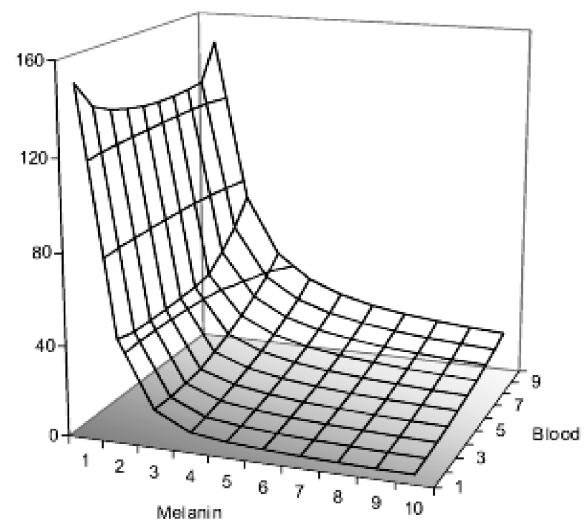

(a)
Optimal filters

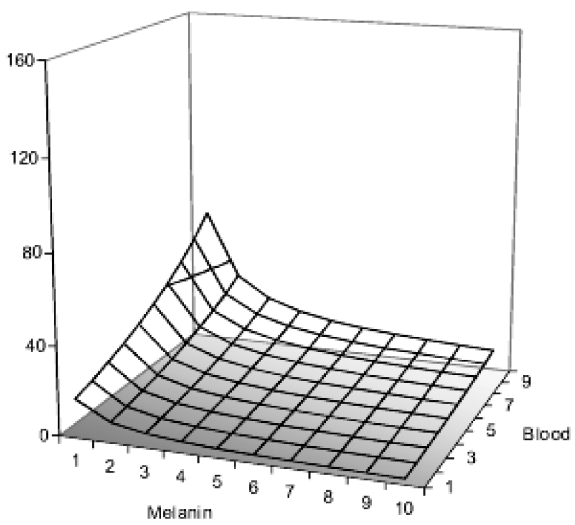

(b)

Fig. 5. Plot of the relative error in the recovery of melanin concentration as a function of melanin and blood concentration, (a) for RGB filters and (b) for the optimal filters.

RGB filters

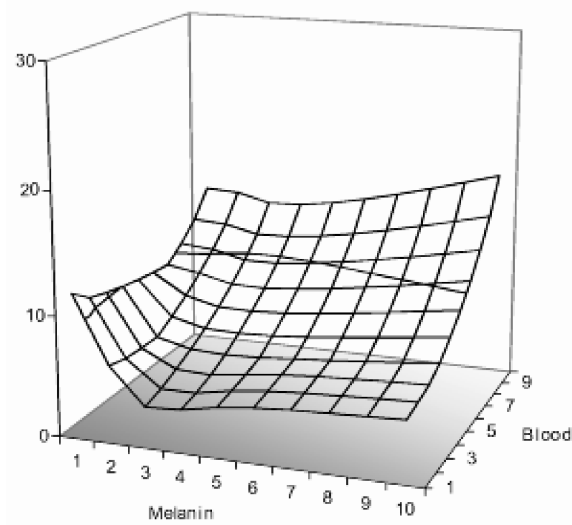

(a)
Optimal filters

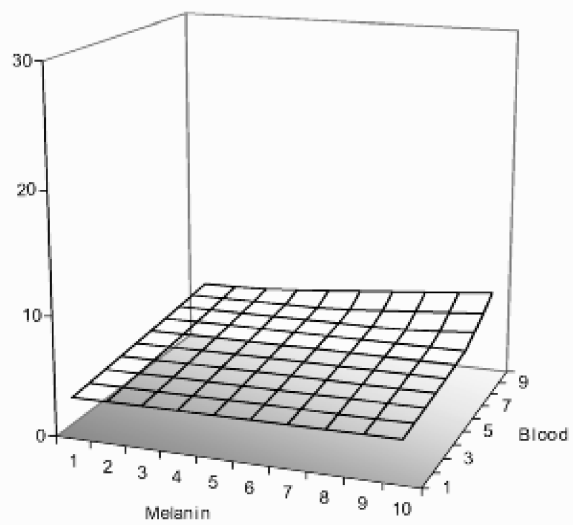

(b)

Fig. 6. Plot of the relative error in the recovery of the thickness of the papillary dermis as a function of melanin and blood concentration, (a) for RGB filters and (b) for the optimal filters.

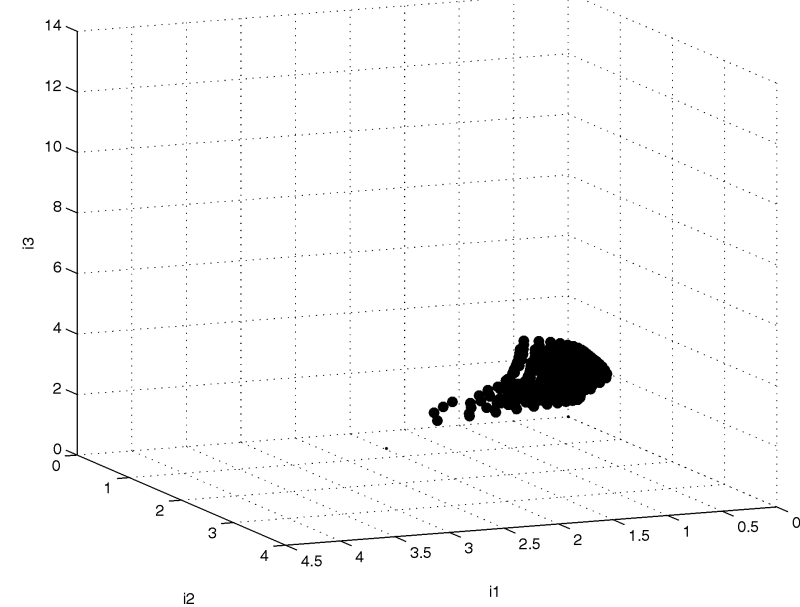

(a)

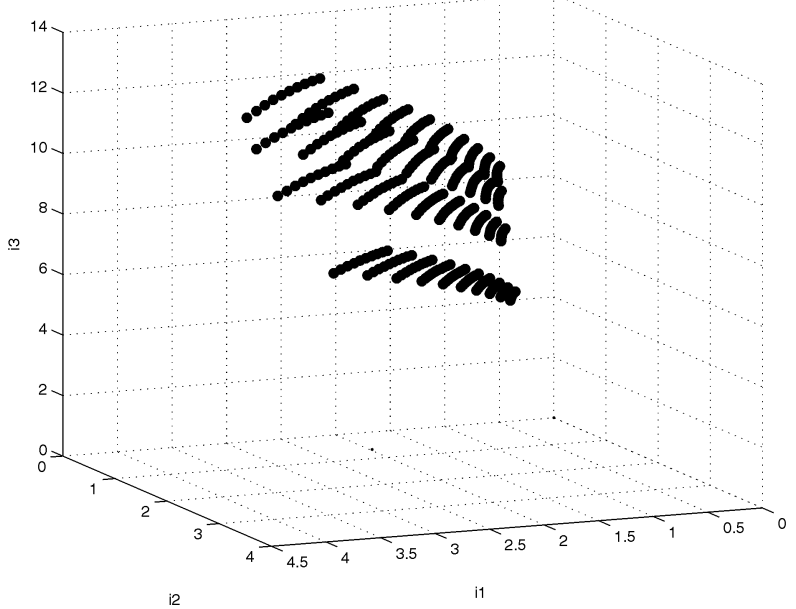

(b)

Fig. 7. Graphical representation of the model of coloration. The box axes represent values of image vectors. The dots represent corresponding parameter values for melanin, blood, and papillary dermis for (a) RGB filters and (b) optimal filters. 
method of tissue quantification, it can be applied within other schemes. For example, by changing the optimization criterion one could compute filters that lead to clustering of parameters corresponding to a given object class, thus enabling their classification.

The method presented here can be related to other methods of skin characterization by considering the characteristic absorption and scatter curves of melanin, blood, and dermis to play the role similar to the basis functions. The quantitative parameters then play the role of coefficients. If only absorption is taken into account, the remitted spectra and, subsequently, the image values, could be expressed as linear combinations of the log functions of the spectra (see (15)). If scatter is also considered, it is necessary to use nonlinear functions. Most of the published methods discussed in Section 2 do not take into account the scatter by collagen and this could be the reason why those methods require more than three basis functions.

It is interesting also to compare our technique to the principal component analysis (PCA) used by a number of researchers, as discussed in Section 2. Using PCA, it is possible to determine the best linear combination of wavelengths (that is of filter response function) with which to measure a scene parameter. Unlike the methodology presented in Sections 4 and 5, PCA assumes independence of the parameters characterizing the data. If this is not the case, as with the skin, then PCA will not necessarily give accurate results. This is because it is not possible to study the effects of a single scene parameter on the remitted spectrum in isolation from other scene parameters. As discussed above, the camera measurement at each image location is defined as a vector valued function of a vector variable; hence, the analysis is valid for any number of scene parameters.

The primary limitation of the proposed technique is that it requires a training set of reflectance spectra a priori, for every possible parameter combination. This must be obtained from either an experimental measurement or via a mathematical model. If a model is required then optical properties for all constituents must be available across the appropriate range of wavelengths along with appropriate ranges of thickness of each of the different layers. The constancy of the illuminating light will impose another restriction. For many applications, such as industrial quality control and medical imaging, it may be possible to ensure a constant illumination source. For other applications, for example, imaging a scene illuminated with natural light, some form of color consistency technique will be required. In some applications, changes in image values corresponding to changes in a particular parameter may not be detectable with a standard digital camera due to its limited brightness sensitivity. This will either need to be accepted as a limitation or a more accurate image acquisition system employed.

The success of the overall methodology has been demonstrated on a three-parameter model of human skin. It has been shown that an objectively defined set of filters would perform better than an RGB system. In practical terms, the RGB filters already give good results, see for example, results in [4] and parametric maps in Fig. 1. Images have not yet been taken with the optimal filters to enable comparison.

\section{Conclusions and Further Work}

This work has demonstrated that the use of spectral filters optimized for specific tissue models leads to a substantial increase in the accuracy with which the parameters characterizing the tissue can be recovered. Provided that the optical characteristics of the tissue components and their laminar structure are known, it is possible to establish both the existence of and the specification for the optimal filters. The technique has a potential for application in other areas of medical imaging and beyond, wherever variations in spectral characteristics of objects of interest can be described by a small number of parameters. Parametric maps showing parameter variations across the image scene are proving to be of considerable value to clinicians in their diagnosis of melanoma.

Work is now underway to apply the methodology to other medical imaging systems. Two potential applications have been identified where it would be of value to clinicians to have a gray-scale representation of histological parameters across the image. The first is the ocular fundus which has been studied in detail by Preece and Claridge [22]. The second is the gastrointestinal tract that is currently under investigation. One major additional challenge in comparison to skin imaging is to apply similar analysis in situation where illumination is spatially uneven and calibration is not easy to perform. Solutions to this problem are being investigated, drawing on algorithms proposed for discounting the illuminant, such as, for example, [23] and [24].

\section{ACKNOWLEDGMENTS}

The authors would like to acknowledge the help and guidance of Dr. Jon Rowe of the School of Computer Science and Mr. Roger Holder of the Department of Mathematics and Statistics at the University of Birmingham.

\section{REFERENCES}

[1] S. Wan, R.R. Anderson, and J.A. Parrish, "Analytical Modeling for the Optical Properties of the Skin with In Vitro and In Vivo Applications," Photochemistry and Photobiology, vol. 34, pp. 493-499, 1981.

[2] S. Cotton and E. Claridge, "Developing a Predictive Model of Human Skin Coloring," Proc. SPIE Medical Imagining, vol. 2708, pp. 814-825, 1996.

[3] E. Angelopoulou, R. Molana, and K. Danilidis, "Multispectral Skin Color Modelling," Proc. IEEE Conf. Computer Vision and Pattern Recognition, pp. 635-642, 2001.

[4] M. Moncrieff, S. Cotton, E. Claridge, and P. Hall, "Spectrophotometric Intracutaneous Analysis: A New Technique for Imaging Pigmented Skin Lesions," British J. Dermatology, vol. 146, pp. 448-457, 2002.

[5] E. Claridge and S. Preece, "An Inverse Method for the Recovery of Tissue Parameters from Color Images," Information Processing in Medical Imaging, pp. 306-317, 2003.

[6] N. Tsumura, H. Haneishi, and Y. Miyake, "Independent Component Analysis of Skin Color Image," J. Am. Optical Soc., vol. 6, no. 9, pp. 2169-2176, 1999.

[7] G. Finlayson and G. Schaefer, "Solving Color Constancy Using a Constrained Dichromatic Reflection Model," Int'l J. Computer Vision, vol. 42, no. 3, pp. 127-144, 2001.

[8] M.A.H. Stoerring and E. Granum, "Physics-Based Modelling of Human Skin under Mixed Illuminants," J. Robotics and Autonomous Systems, vol. 35, pp. 131-142, 2001.

[9] T. Ohtsuki and G. Healey, "Using Color and Geometric Models for Extracting Facial Features," The J. Imaging Science and Technology, vol. 42, no. 6, pp. 554-561, 1998. 
[10] S.A. Prahl, M. Keijzer, S.L. Jacques, and A.J. Welch, “A Monte Carlo Model of Light Propagation in Tissue," SPIE Inst. Series IS, vol. 5, pp. 102-111, 1989.

[11] P. Kubelka and F. Munk, "Ein Beitrag zur Optik der Farbanstriche," Zeitschrift fur Technishen Physik, vol. 11, pp. 593-611, 1931.

[12] S. Arridge and J. Hebden, "Optical Imaging in Medicine: II Modelling and Reconstruction," Physics in Medicine and Biology, vol. 42, pp. 841-854, 1997.

[13] C. Chang, Q. Du, T. Sun, and M. Althouse, "A Joint Band Prioritisation and Band-Decorrelation Approach to Band Selection for Hyperspectral Image Classification," IEEE Trans. Geoscience and Remote Sensing, vol. 37, no. 6, pp. 2631-1641, 1999.

[14] S. Shen and E. Bassett, "Information Theory Based Band Selection and Utility Evaluation for Reflective Spectral Systems," SPIE Proc. Algorithms and Technologies for Multispectral, Hyperspectral and Ultraspectral Imagery VIII, S.S. Shen and P.E. Lewis, eds., vol. 4725, pp. 18-29, 2002.

[15] J. Karlholm and I. Rehnorn, "Wavelength Band Selection Method for Multispectral Target Detection," Applied Optics, vol. 41, no. 32, pp. 6786-6795, 2002.

[16] J. Price, "An Approach for Analysis of Reflectance Spectra," Remote Sensing of Environment, vol. 64, no. 3, pp. 316-330, 1998.

[17] S. Cotton and E. Claridge, "Noninvasive Skin Imaging," Information Processing in Medical Imaging, vol. 1230, pp. 501-507, 1997.

[18] M.M. Lipschutz, Differential Geometry. New York: McGraw-Hill Book Company, 1969.

[19] L. Lyons, Statistics for Nuclear and Particle Physicists. Cambridge: Cambridge Univ. Press, 1986.

[20] M.G. Kendall and A. Stuart, The Advanced Theory of Statistics. Volume 1: Distribution Theory, third ed. London: Charles Griffin \& Co. Ltd., 1969.

[21] R. Anderson and J. Parrish, "The Optics of Human Skin," The J. Investigative Dermatology, vol. 77, no. 1, pp. 13-19, 1981.

[22] S.J. Preece and E. Claridge, "Monte Carlo Modelling of the Spectral Reflectance of the Human Eye," Physics in Medicine and Biology, vol. 47, pp. 2863-2877, 2002.

[23] G. Healey and D. Slater, "Global Color Constancy: Recognition of Objects by Use of Illumination Invariant Properties of Color Distributions," J. Optical Soc. Am., vol. 11, no. 11, pp. 3003-3010, 1994.

[24] L. Maloney and B. Wandell, "Color Constancy: A Method for Recovering Surface Spectral Reflectance," J. Optical Soc. Am., vol. 3, no. 1, pp. 29-33, 1986.

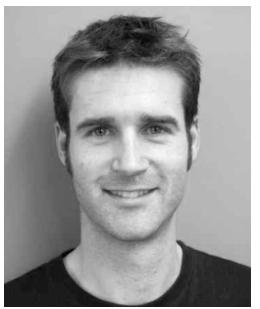

Stephen J. Preece received the BSc Hons degree in physics with theoretical physics in 1995 and the PhD degree in applied mathematics in 1999, both from the University of Birmingham, United Kingdom. He then worked as a postdoctoral researcher in the Computer Science Department at Birmingham and is now working for Astron Clinica as a research scientist. His current interests include the interpretation of medical images based on the physics of image formation, the computational modeling, and biomechanics.

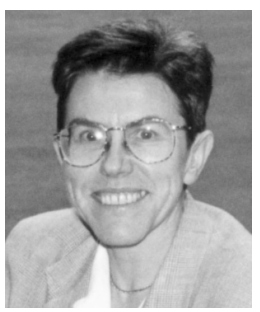

Ela Claridge received the MSc degree in mathematics with computer science in 1978 from Gdansk University, Poland, and the PhD degree in computer science in 1984 from the University of Birmingham, United Kingdom. She worked as a lecturer at Aston University until 1989 and then joined the School of Computer Science at the University of Birmingham where she is now a Reader. She leads an interdisciplinary research group specializing in medical image interpretation. She is a Fellow of the British Computer Society, a Chartered Engineer, and a Member of the British Machine Vision Association. Her current research interests include the physics of image formation, modeling of human visual perception, and evolutionary computation for computer vision.

$\triangleright$ For more information on this or any other computing topic, please visit our Digital Library at www.computer.org/publications/dlib. 